\title{
The Design of Coulometric Thickness Gauge Based on Microprocessor
}

\author{
Limin Cai \\ School of physics and information engineering, Jianghan \\ University, Wuhan, Hubei, China \\ cailm@aliyun.com \\ Song Tang* \\ Graduate faculty, Jianghan University, Wuhan, Hubei, \\ China \\ tangsng@live.cn
}

\begin{abstract}
In order to solve the issues of low accuracy, existing electrolytic coating thickness, poor reproducibility, lack of self-checking, data is difficult to store and handle urgent need to address, we designed a portable gauge based on Coulomblaw. This paper describes the system design of electrolytic thickness, the hardware and software design. Gauge meets factory testing needs through a variety of different materials, coatings extensive experiments.
\end{abstract}

Keywords-Single-chip microprocessor; gauge; numerical control constant current source

\section{INTRODUCTION}

Electrolytic thickness widely used shipbuilding, petrochemicals, power plants, automobile manufacturing, machinery manufacturing industry which can measure the hull, deck, boils, pipes, tanks, rail, slab casting, the thickness of machining parts, analysis the corrosion degree[1]. Therefore the use of gauge has made great contributions to the entire economy and development of electroplating industrial.

With the improvement of user demand, the rapid development of electronics industry, the existing instruments already slightly out of data, there are many weaknesses as follows:

1) The accuracy about coating measurement is not enough to meet precision coating requirements;

2) The stability is not high, there will be a large error when repeated measurements;

3) The pressure between electrolyze and plating, aging circuit causes constant current source change;

4) The lack of a constant current calibration function;

5) Data is difficult to store, process and other urgent issues.

This project designed a portable electrolytic thickness based on MSP430 microcontroller, which easy to operate with a small volume and other characteristics, achieve a precise measurement, but also have a programmable function that can store large amounts of information, overcome existing gauge issues.

\author{
Shizhen Zhang \\ Graduate faculty, Hubei University of Technology, Wuhan, \\ Hubei, China \\ zsz108@live.cn \\ Wu Xia \\ Graduate faculty, Jianghan University, Wuhan, Hubei, \\ China \\ xiawuwhjd@163.com
}

\section{SYSTEM DESIGN}

Coulomb electrolytic method is based on using a constant direct current to make anodic dissolution of the coating, and by calculating consumed time to measure coating thickness. Measuring range is generally 0-50 um, ensuring accuracy, the thicker the coating can also be measured, the errorwill gradually become larger. Indication error is less than equal to $\pm 10 \%$, and the resolution of gold, decorative chrome is $0.01 \mathrm{um}$, other coating types is $0.1 \mathrm{um}$.

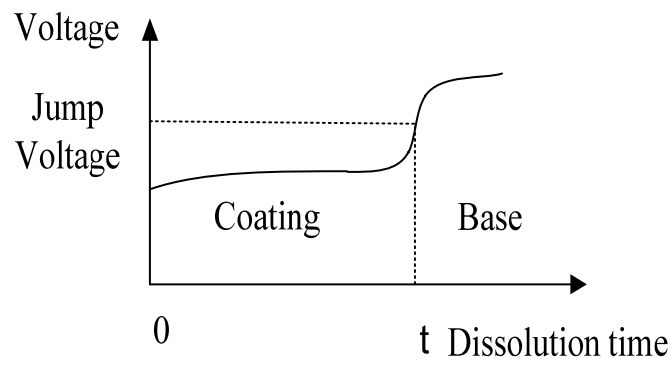

Figure 2-1. Voltage-Dissolving time graph showing

Shown in Figure 2-1, the figure curve is voltage across the cell charts over time in the electrolysis process, time $\mathrm{t}$ for a "transition voltage", gauge will convert time t into coating corresponding thickness. The coating thickness can be calculated according to the following equation (1). If the anode solubility coefficient $\mathrm{v}$ is constant, the metal coating is only proportional to the reaction time $t$.

$$
\mathrm{d}=\mathrm{v} \cdot \mathrm{t}
$$

$\mathrm{t}-$ Electrolysis reaction time; v-Anodic dissolution coefficient

The $\mathrm{v}$ relate with reaction temperature, electrolyte concentration, anodic dissolution by the cell area and current size. Several other parameters is constant during the reaction, and $\mathrm{v}$ depend on current only, so the value of $\mathrm{v}$ can be obtained through the corresponding standard sheet metal calibration measurement, and the coating of each converted into the corresponding a constant current value. So that coating thickness can be obtained as long as measurement time of electrolysis reaction. 
The key is to determine reaction time $t$, the electrolysis reaction is usually designed using a small stainless steel cell, and install a rubber ring in the bottom of the cell, the hole is used to determine the measurement ring (anode dissolution) in the area to for the end of the plating cell, the cell was added an appropriate electrolyte as the cathode for electrolysis of the sample as the anode, with a constant DC current through the electrolytic bath and the plating, the coating has been dissolved. The current coating dissolves when exposed sub-layer metal finish, the instrument will detect changes in the electrolysis voltage, the voltage value at this transition voltage, the time spent for the reaction time $\mathrm{t}[2]$.

As discussed principle above we can know parameters that influence reaction time $t$, respectively, constant current source value, the size of the apron, motor speed, so the design of the system must provide convenience to user set these parameters, and require these parameters can be powered down maintained.

\section{HARDWARE DESIGN}

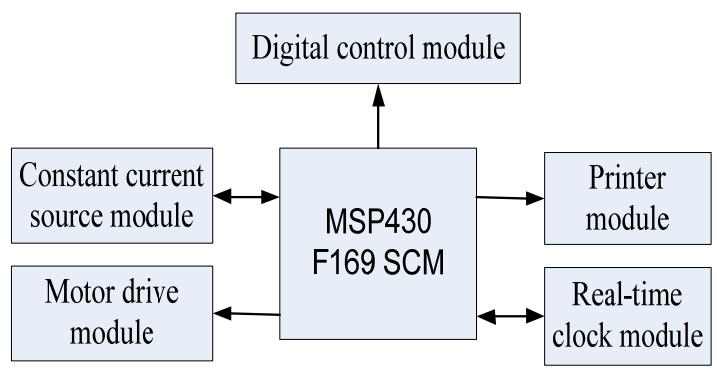

Figure 3-1. Schematic diagram of hardware

Our design uses MSP430F169 as master microcontroller with DA output functions, also mainly contains the printer module, LED display module, the constant current source module, key module. Single chip control the entire system, and constant current source circuit is important to make gauge theory of electrolytic realized. Digital display module displays measured thickness and voltage, key is to control microcontroller input module, while introduce real-time clock for easy to use. This design uses DALLAS DS1302-based company launched a real-time clock circuit, with low power, comes with RAM, and can be years, months, days, hours, minutes and seconds for timing, but also has leap year compensation function [3]. Figure 3-1 showsthe hardware schematic structure of electrolytic thickness.

\section{A Single-chip control module}

Measure the performance of a single-chip index is:fast operation, low price, low power consumption, powerful and high stability [4]. TI's MSP430 series is a special emphasis on low-power single-chip varieties. This series has a lot of models, they are made by some of the basic functions of the application modules in different target combinations.
Sampling 16 MSP430F169 Series CPU RISC systems, 125ns instruction cycle, integrated with 16 registers, and constant generator, play the highest code execution efficiency. There are two 16-bit timers, two serial communication ports, 6-channel 12-bit A / D with a sample and hold, dual 12-bit D / A synchronous conversion, 60KB $+256 \mathrm{~B}$ flash memory $2 \mathrm{~KB}$ RAM, there is a very critical with programmable supply voltage level detection manager / monitor, which provides a convenient way for our design gauge without modifying the current value of the corresponding current source material through the programming process, and saved to the flash power down.

\section{B Constant current source circuit}

Constant current source circuit is the core part of the whole electrolytic thickness hardware design, directly affect the stability and accuracy of coating thickness measurement. This design uses a circuit schematic shown as 3-2-1 is MSP430F169 voltage output DA, through the conversion circuit, the output of numerical control constant direct current output accuracy is determined by the microcontroller $12 \mathrm{DA}$ decision. Where is the microcontroller DA output current through need for constant DC current, is a current feedback sampling resistor, is the load resistor, R5 for the current limiting resistor.

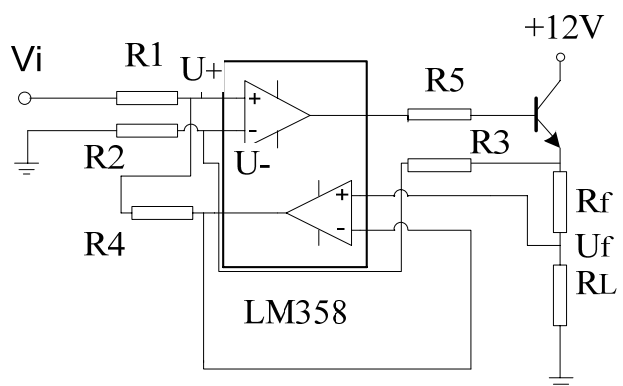

Figure 3-2-1. Numerical constant current source circuit

$$
\begin{gathered}
\frac{\mathrm{V}_{\mathrm{i}}-\mathrm{U}_{+}}{\mathrm{R}_{1}}=\frac{\mathrm{U}_{+}-\mathrm{U}_{\mathrm{f}}}{\mathrm{R}_{4}} \\
\mathrm{U}_{\mathrm{f}}=\mathrm{I} \cdot \mathrm{R}_{\mathrm{L}} \\
\mathrm{U}_{+}=\mathrm{U}_{-}=\frac{\mathrm{R}_{2}}{\mathrm{R}_{2}+\mathrm{R}_{3}} \cdot \mathrm{I} \cdot\left(\mathrm{R}_{\mathrm{f}}+\mathrm{R}_{\mathrm{L}}\right)
\end{gathered}
$$

If we take $\mathrm{R} 1=\mathrm{R} 2=\mathrm{R} 3=\mathrm{R} 4$, the current can be calculated by the equation (2), (3), (4):

$$
\mathrm{I}=\mathrm{V}_{\mathrm{i}} / \mathrm{R}_{\mathrm{f}}
$$

From the above we can know, the current I through the load is determined only by the input voltage, and the resistance value is independent of the size of load, but due to limitations of the operationalamplifier, only load change within a certain range, the current through the load is constant.

For MSP430F169 the DA is 12 resolutions, we can calculate that this numerical minimum adjustable constant 
current source circuit current is $0.015 \mathrm{~mA}$, to meet the actual measurement needs.

\section{SOFTWARE DESIGN}

For single-chip, software design related to the success or failure about a system. The gauge uses $\mathrm{C}$ language to design system program which can improve the code portability, readability and maintainability. The modular program design ,including main program, digital display unit, A/D sampling unit, D/A constant current source, the motor unit, printer unit, real-time clock unit. The flow chart of main program as $4-1$.

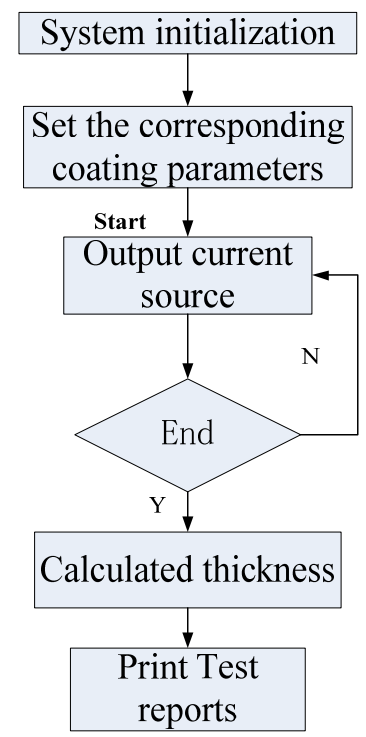

Figure 4-1. Main program flow chart

After electrolysis gauge power, initialize program in the first. Then select material, the size of rubbergasket(electrolysis reaction area), when you press the "Start"key gauge begin working the end of reaction. Digital tube display thickness, and save the data.

\section{A Digital tube display module}

This design uses two sets of digital tube and every set has four. The first set is to display coating thickness, and can display the detected voltage value through the key switch; second group is to display real-time clock, though the key can switch sensitivity, constant current source value, motor speed and other parameters are displayed.

\section{B Printer module}

In order to make the gauge easy to use, we joined the $5 \mathrm{~V}$ printer. Some important information can be stored in the microcontroller to print out, such as gauge models, coating name, coating thickness, measurement time.

\section{Power-down save parameters}

MSP430F169 microcontroller has flash self-programming function, so it can save the value of a constant current source, motor speed, sensitivity and other parameters to modify directly, and also supports to add user-defined new material coating, corresponding sensitivity, do not modify and download the program again.

\section{SUMMARY}

This gauge has following advantages through practical application:

1) The hardware circuit design becomes simple, easy production, low cost, because use MSP430 as the controller;

2) The output of current source accuracy can reach $0.015 \mathrm{~mA}$, provides more possible to measuring coating material;

3) Constant current source can be self-checking, in order to avoid prolonged use, temperature or other environment parameters change lead to current deviation occurs;

4) MSP430 MCU flash has 60KB, fully satisfy related coating material parameters and the historical measurement results long-term preservation.

In 21st century artificial intelligence technology has a rapid development. Surface treatment of various areas applies computer technology to control the production process. With automated rapid development, it should be singled-chip microprocessor and coulomb thickness measurementtechnology combine to develop new electrolytic thickness [5]. This gauge has high accuracy based on MSP430F169 design, stability, fast speed, low cast, and MSP430 MCU enjoy lower power features that provides good scalabilityfor handheld gauge in the future.

\section{ACKNOWLEDGEMENTS}

This work was financially supported by Wuhan Science and Technology research project(201250499145-23) and the project of production-study-research in Wuhan city-belonged university(cxy04).

\section{CORRESPONDING AUTHOR}

Name: Song Tang, Email: tangsng@live.cn, Mobile phone: 18271396980

\section{REFERENCES}

[1] Zanyuan Wang. Development of portable ultrasonic thickness gauge based on microcontroller. Beijing Chemical Technology University (2011).

[2] Commonly used coating thickness test instrument-Electrolytic thickness lectures.Surface Engineering Consulting.59-62(2012).

[3] Tianxiang Guo.51 microcontroller C language tutorial.Beijing: Electronic Industry Press.323-331(2008).

[4] Jianhua Shen, Yanqin Yang.MSP430 series 16 ultra-low power microcontroller principles and practice.Beijing: Beijing University of Aeronautics and Astronautics Press.2-15(2008).

[5] JianguoLiu, Coulomb electrolytic thickness 30-year review of application, Plating and finishing.47-48(2011).

[6] Rick Greyhen,RobertMoote,IngoCyliax. Real-Time Programming. Beijing: China Electric Power Press(2001). 